\section{SCIENCE CHINA \\ Physics, Mechanics \& Astronomy}

-Editorial•

Editor's Focus
April 2021 Vol. 64 No. 4: 240361

https://doi.org/10.1007/s11433-021-1669-4

\title{
Electro-optic modulation using lithium niobate metasurfaces
}

Dynamic control of optical fields using metasurfaces has remained a hot pursuit over the past decades. Such control requires the tuning of light-matter interactions in the ultrathin layers of materials. The established approaches to dynamically alter the optical response of metasurfaces include optical pumping, thermal heating, chemical reaction, and electrical stimulation [1]. Among all these tuning methods, electrical tuning is of particular interest, because it holds promise to integrate metasurfaces with on-chip optoelectronic devices.

Lithium niobate $\left(\mathrm{LiNO}_{3}, \mathrm{LN}\right)$ could be a promising material to satisfy the control requirement. As its refractive index can be changed by applying an electrical voltage, $\mathrm{LN}$ has become a workhorse for the modulators in modern communication systems to encode optical waves according to external electric signals. However, owing to the absence of optical constraints in the direction normal to the surface, traditional bulk LNs are not proper for optical field confinement and control at the nanoscale. Of note, the emerging thin-film LN on insulator (LNOI) has brought light to this dilemma. The large refractive index contrast between the LN film and substrate enables tight localization of optical modes within the nanometres-thin LN layer, thus offering an ideal framework for the fabrication of the LN nanostructures with tunable properties [2-4].

The accompanying paper entitled "Electro-optic lithium niobate metasurfaces" by Gao et al. [5] reports the design and fabrication of an LN metasurface that provides EO phase modulation to transmitted light. By leveraging the quasi-bound-statesin-the-continuum inside the metasurface array, the EO effect induced by an applied bias voltage is significantly enhanced compared with the unstructured LN film. An obvious phase modulation is also observed. This finding would pave the way toward the development of spatial light modulators in wavefront engineering, pulse shaping, polarization control, etc.

GuiLu Long

Department of Physics, Tsinghua University, Beijing 100084, China

1 A. M. Shaltout, V. M. Shalaev, and M. L. Brongersma, Science 364, eaat3100 (2019).

2 L.-K. Chen, and Y.-F. Xiao, Sci. China-Phys. Mech. Astron. 63, 224231 (2020).

3 M. Wang, R. Wu, J. Lin, J. Zhang, Z. Fang, Z. Chai, and Y. Cheng, Quantum Eng. 1, e9 (2019).
4 M. He, M. Xu, Y. Ren, J. Jian, Z. Ruan, Y. Xu, S. Gao, S. Sun, X. Wen, L. Zhou, L. Liu, C. Guo, H. Chen, S. Yu, L. Liu, and X. Cai, Nat. Photon. 13, 359 (2019), arXiv: 1807.10362.

5 B. F. Gao, M. X. Ren, W. Wu, W. Cai, and J. J. Xu, Sci. China-Phys. Mech. Astron. 64, 240362 (2021). 